\title{
Introdução do coeficiente de uniformidade para avaliação de revestimentos asfálticos do tipo tratamentos superficiais
}

\author{
Raimi Costa da Silva ${ }^{1}$, Suelly Helena de Araújo Barroso², Youngsoo Richard Kim ${ }^{3}$ \\ 1Universidade Federal do Ceará, UFC, Programa de Engenharia de Transportes, PETRAN, raimi@det.ufc.br \\ ${ }^{2}$ Universidade Federal do Ceará, UFC, Programa de Engenharia de Transportes, PETRAN, suelly@det.ufc.br \\ ${ }^{3}$ North Carolina State University, NCSU, Departamento of Civil, Construction, and Environmental Engineering, kim@ncsu.edu
}

\section{Recebido:}

4 de maio de 2017

Aceito para publicação:

9 de novembro de 2017

Publicado:

30 de abril de 2017

Editor de área:

Francisco Thiago Aragão

\section{Palavras-chaves:}

Tratamento superficial por penetração;

Defeitos;

Perda de agregado;

Coeficiente de uniformidade.

\section{Keywords:}

Surface treatments;

Distresses;

Aggregate loss;

Uniformity coefficient.

DOI:10.14295/transportes.v26i1.1371

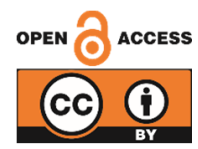

\begin{abstract}
RESUMO
O Tratamento Superficial por Penetração (TSP) é o revestimento mais empregado no Brasil em Rodovias de Baixo Volume de Tráfego. Após investigações de campo e de laboratório, observou-se que os principais defeitos dos TSPs são a perda de agregados e a exsudação que podem comprometer o seu desempenho, bem como aumentar os seus custos. No Brasil, ainda existem poucos estudos de dosagem e de avaliação dos TSPs na fase de laboratório para mitigar esses dois defeitos. Sendo assim, investigaram-se metodologias americanas como as utilizadas na North Carolina State University e verificouse que o coeficiente chamado de PUC (Performance-Based Uniformity Coefficient) é um parâmetro associado à distribuição granulométrica do agregado que apresenta potencial de prever a exsudação e a desagregação desse tipo de revestimento. Concluiu-se que o PUC é um excelente parâmetro para ser introduzido em procedimentos de dosagem de TSP no Brasil e que as faixas recomendadas pelo DNIT para TSP devem ser revistas.
\end{abstract}

\begin{abstract}
The surface treatments are the most used type of coating in Brazil in Low Traffic Volume Roads, more specifically the chip seal. After field and laboratory investigations, it was observed that the main defects of chip seals are aggregate loss (or raveling) and bleeding, which can affect its performance and increase considerably the paving costs. In Brazil there are still few studies of design and performance of chip seals in the laboratory phase to predict these two defects. Thus, it was investigated materials and methodologies employed at North Carolina State University and it was verified that the coefficient called Performance-Based Uniformity Coefficient (PUC) is a parameter associated with the granulometric distribution of the aggregate that has potential to predict bleeding and raveling of this type of coating. It was concluded that PUC is an excellent parameter to be introduced in chip seal design procedures and that the ranges recommended by DNIT to chip seals should be reviewed.
\end{abstract}

\section{INTRODUÇÃO}

A Confederação Nacional do Transporte (CNT) relatou na pesquisa de rodovias de 2016 que o Brasil apresenta $1.351 .979 \mathrm{~km}(78,6 \%)$ de rodovias não pavimentadas, $211.468 \mathrm{~km}(12,3 \%)$ de rodovias pavimentadas e $157.309 \mathrm{~km}$ de rodovias planejadas (9,1\%). Tendo em vista esse cenário, percebe-se que a falta de vias de acesso pavimentadas pode provocar inúmeros problemas à população, tais como: a falta de itens de necessidade básica como saúde e educação, isolamento, entraves econômicos e até comprometimento da qualidade de vida.

Uma solução econômica para a ampliação da malha pavimentada brasileira seria o melhor emprego de revestimentos delgados em Rodovias de Baixo Volume de Tráfego (RBVTs), como Tratamento Superficial por Penetração (TSP). Adotou-se aqui a nomenclatura Tratamento Superficial por Penetração para 
o tipo de revestimento esbelto construído pela aplicação sucessiva de ligante e agregado, seguida de compactação (Larsen, 1985). Além do seu caráter econômico, o TSP é considerado uma técnica de fácil aplicação, com rápida execução e liberação da pista (Nogami e Villibor, 1995). Internacionalmente, o TSP é uma técnica largamente utilizada para a manutenção preventiva e corretiva de pavimentos em rodovias de médio e alto volume de tráfego (Lee e Kim, 2008a).

Pesquisas indicam que o TSP é o principal revestimento empregado no estado do Ceará, representando 61\% da malha rodoviária pavimentada (DER-CE, 2014). Em contraponto, notou-se que há poucos estudos voltados para TSPs no Brasil e que não existem métodos de laboratório/campo para avaliação dos seus principais defeitos. A partir de estudos bibliográficos como os de: Loiola (2009); Lee e Kim (2008a, 2008b, 2009, 2010); Pereira (2011, 2013); Adams (2014); Silva (2016), e de observações de campo, comprovou-se que os defeitos mais críticos dos TSPs são a perda de agregado e a exsudação.

Loiola (2009) introduziu em seu estudo uma adaptação do ensaio Wet Track Abrasion Test (WTAT), realizado para Microrrevestimento Asfáltico a Frio (MRAF), a fim de avaliar a perda de agregado para TSP. Pereira $(2010,2013)$ utilizou o mesmo princípio e adaptou o método proposto por Loiola (2009) realizando um processo de compactação diferente e ensaiando também o TSP em um simulador de tráfego de pequeno porte.

Pesquisas desenvolvidas no estado da Carolina do Norte/USA apresentam diferentes formas de avaliar o desempenho do TSP. Dessa forma, optou-se por introduzir no contexto nacional brasileiro um parâmetro chamado PUC (Performance-Based Uniformity Coefficient) idealizado por Lee e Kim (2009), o qual fornece uma previsão aproximada do percentual de exsudação e da perda de agregado no revestimento avaliando a curva granulométrica do agregado empregado.

O empirismo na dosagem do TSP, a falta de equipamentos adequados para a avaliação de desempenho e a escassez de pesquisas nacionais foram os principais motivadores do estudo aqui proposto. A correta construção do TSP e a utilização de uma dosagem adequada poderiam gerar maior aproveitamento dos materiais naturais, bem como viabilizar o emprego de materiais alternativos em substituição parcial ao agregado natural, o que diminuiria o passivo ambiental advindo deste serviço. Tal cenário mostra a relevância do tema para a ampliação e a melhoria da malha rodoviária brasileira, necessitando, portanto, de maior atenção por parte dos órgãos reguladores e do meio científico.

O principal objetivo desta pesquisa foi introduzir no contexto nacional o coeficiente PUC, ainda não divulgado nos trabalhos brasileiros, para análise do desempenho dos tratamentos superficiais por penetração a partir dos estudos realizados nos estados do Ceará/Brasil e da Carolina do Norte/EUA. Dada a importância da uniformidade do agregado comprovada pela comunidade internacional (Lee e Kim, 2009; Transit in New Zealand, 2005), investigou-se como esse conceito é abordado nas normas técnicas nacionais e como pode ser relacionado com os estudos de laboratório atualmente desenvolvidos no Brasil.

\section{BREVE REVISÃO BIBLIOGRÁFICA}

\subsection{Defeitos dos tratamentos superficiais por penetração}

A perda de agregado e a exsudação são os dois defeitos mais severos encontrados em TSPs (Lee e Kim, 2008a). De acordo com Lee e Kim (2008a) existem fatores intrínsecos que afetam a adesão entre o ligante e o agregado nos TSPs, como porosidade, área de superfície, capacidade de absorção, mineralogia e textura da superfície do agregado, o tipo de emulsão e o seu tempo de ruptura.

Adams (2014) relata que as maiores perdas de agregado ocorrem logo durante a passagem inicial do tráfego e as suas principais causas estão associadas: ao tempo chuvoso, à taxa excessiva de agregados, ao controle de tráfego inadequado, à quantidade de ligante inadequado para recobrir o agregado, as condições impróprias do agregado (sujos, com presença de finos), entre outros. Além desses fatores, sabe-se que a coordenação dos equipamentos na construção é essencial para evitar a ruptura precoce da emulsão. 
Além da perda de agregado precoce do TSP, Moraes e Bahia (2013) verificaram a sua evolução durante toda a vida do pavimento. Uma hipótese levantada é de que a perda precoce ocorre principalmente devido à ligação entre a emulsão e o agregado ainda não ser forte o suficiente para resistir ao tráfego. Já a perda de agregado tardia poderia acontecer devido ao envelhecimento oxidativo que confere menor flexibilidade ao revestimento, tornando-o menos tolerante às deformações. Essa perda tardia, ao contrário da precoce, tende a aumentar com o passar do tempo, pois o revestimento se torna mais rígido.

Devido à significativa utilização do TSD (Tratamento Superficial Duplo) em rodovias do estado do Ceará, Loiola (2009) investigou os principais defeitos observados em rodovias com esse tipo de revestimento. Adams (2014) também observou os principais defeitos encontrados em TSPs de rodovias do estado da Carolina do Norte e foram percebidas muitas semelhanças com o estudo de Loiola (2009), como pode ser observado nas Figuras 1 e 2.

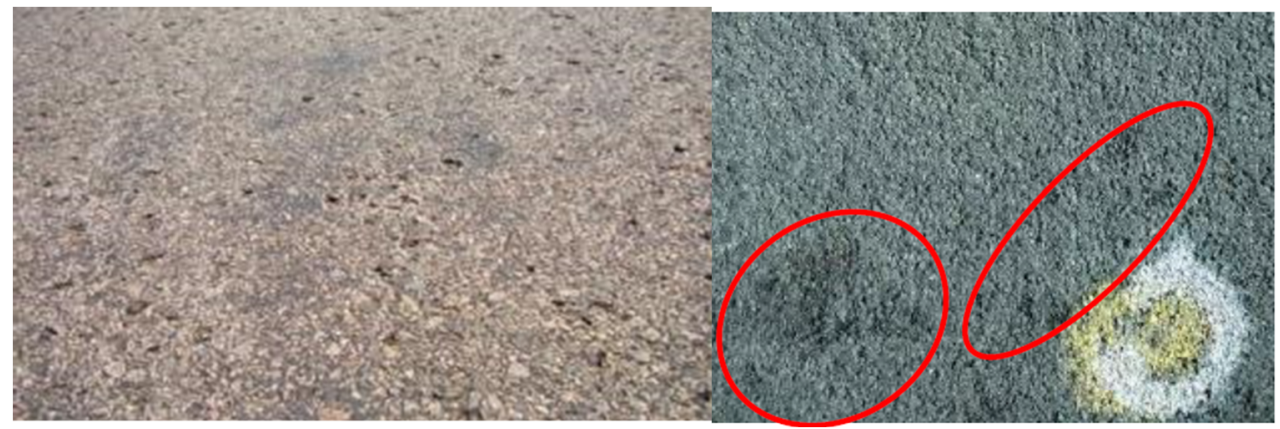

(a)

(b)

Figura 1: Perda de agregado nos estados do Ceará/Brasil e Carolina do Norte/USA, (a) Ceará (Loiola, 2009), (b) Carolina do Norte (Adams, 2014)

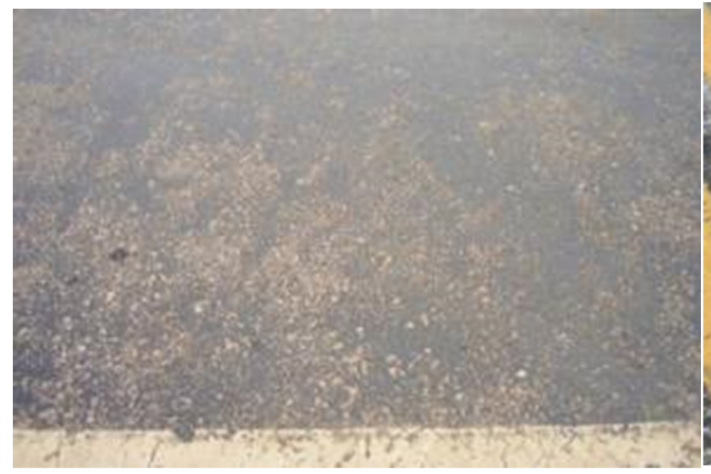

(a)

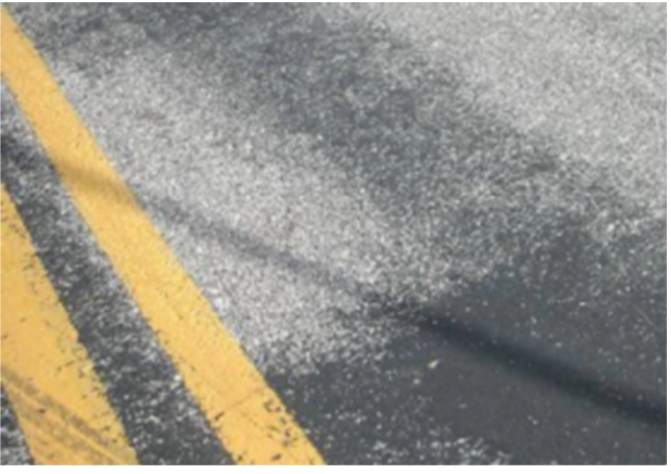

(b)

Figura 2: Exsudação nos estados do Ceará/Brasil e Carolina do Norte/USA, (a) Ceará (Loiola, 2009), (b) Carolina do Norte (Adams, 2014)

\subsection{Métodos de dosagem dos TSPs}

O método da caixa dosadora (Larsen, 1985) utiliza uma caixa de dimensões $80 \times 25 \times 4 \mathrm{~cm}$, na qual os agregados são colocados no fundo da caixa disposta horizontalmente. Na sequência, a caixa é posicionada na vertical, fazendo-se a leitura direta a partir da graduação da tampa da caixa e obtém-se a taxa de agregado em $L / \mathrm{m}^{2}$ a ser utilizada. Esse método serviu de inspiração para muitos métodos realizados ainda hoje nas obras rodoviárias.

O método da bandeja ou do mosaico é muito difundido no meio técnico e segue uma filosofia semelhante ao da caixa dosadora, no qual o agregado é disposto uniformemente sobre o fundo de uma bandeja de área $50 \times 50 \mathrm{~cm}$. Ao colocar o agregado, busca-se preencher todos os vazios, evitando a sua sobreposição, de forma que o fundo da bandeja seja totalmente coberto. 
O DER-CE denomina esse método como o da "bandeja de fundo vermelho", no qual o fundo desta é pintado para facilitar a identificação dos vazios e preenchê-los. Ao fim, pesa-se a bandeja com o agregado e a diferença de peso para a bandeja vazia fornece a quantidade de agregado necessário por metro quadrado. As especificações do DER-CE (2000) se baseiam no método de Tagle e Podestá, em que a taxa de agregado é função da granulometria e a taxa de ligante é função da taxa de agregado multiplicada por uma constante que depende do tipo de emulsão utilizada.

Adams (2014) propôs um método de dosagem de TSPs de uma camada, utilizando como critérios de desempenho principais a perda de agregado e a exsudação. No método proposto, realizou-se ensaio de bandeja modificado, estudo da densidade dos agregados, processamento digital de imagens e análise a laser para verificação dos volumes de emulsão, volume de vazios e volume absorvido pela base granular.

\subsection{Métodos de avaliação do desempenho dos TSPs em laboratório}

No Brasil, Loiola (2009) comparou a perda de agregado obtida em campo com o obtido em amostras de laboratório com o Wet Track Abrasion Test. 0 ensaio de WTAT é simplificadamente um ensaio de desgaste usado comumente para dosagem de MRAF, especificado na ABNT NBR 14746/2001. Loiola (2009) e Pereira $(2010,2013)$ adaptaram o ensaio com corpos de prova em forma circular de aproximadamente $27 \mathrm{~cm}$ para verificação do desgaste no TSP.

Pereira (2013) usou um simulador de tráfego de laboratório de pequeno porte do tipo LCPC para determinar a melhor dosagem de TSP, mas considerou que o ensaio WTAT aplicado ao TSP pode ser uma alternativa econômica para avaliar o desempenho deste tipo de revestimento. Entretanto, o autor comentou que devido à granulometria do agregado, o procedimento pode provocar uma abrasão mais severa do que a causada pelo rolamento dos veículos. 0 autor propôs ainda faixas relacionadas ao Desgaste (D) obtido no ensaio WTAT, considerando o desempenho de amostras com $\mathrm{D}<10 \%$ muito bom, com $10 \% \leq \mathrm{D}<20 \%$ bom, amostras com $20 \% \leq \mathrm{D}<30 \%$ regular e $\mathrm{D} \geq 30 \%$ ruim.

Em pesquisas realizadas no estado da Carolina do Norte são empregados diversos ensaios, como o Flip-Over Test que visa avaliar a perda de agregado precoce ocorrente logo após a compactação e liberação do tráfego, pois se entende como sendo este o período em que a ocorrência deste defeito é mais crítica. 0 Flip-Over Test (FOT) é especificado na ASTM D7000 e nesse ensaio posiciona-se a amostra a $90^{\circ}$ da superfície e em seguida varre-se a superfície com uma escova ou um pincel de cerdas macias para simular a retirada do excesso de agregado que é feita em campo (Figura 3a). Esse ensaio já foi aplicado por Lee e Kim (2008a) para verificação do processo de compactação, por Lee e Kim (2008b) para compreensão dos efeitos das taxas de emulsão e de agregado no desempenho do revestimento e por Adams (2014) para o desenvolvimento de um método de dosagem.

Outra abordagem empregada é o Vialit Test (Figura 3b), um ensaio de adesão cuja base do equipamento é uma placa de aço quadrada de $203 \times 203 \mathrm{~mm}$ em que se posiciona a amostra a $180^{\circ}$ da superfície com a face virada para baixo. No ensaio uma esfera de metal de $500 \pm 5 \mathrm{~g}$ é posicionada a $50 \mathrm{~cm}$ da placa da amostra e, para que o ensaio seja válido, a esfera deve cair do repouso três vezes num intervalo de 10 segundos em cima da placa. Após esse processo, a amostra é pesada para verificação da perda de agregado. Esse ensaio já foi aplicado por Lee e Kim (2010) para avaliação da quantidade de passadas na etapa de compactação de um TSP. Em menor escala se utilizam equipamentos como o Third-Scale Model Mobile Loading Simulator (MMLS3), um simulador de carregamento móvel 3D que simula carregamentos de tráfego de campo e avalia o estado da amostra após o carregamento.

Voltado para a avaliação da exsudação, Lee e Kim (2008b) utilizaram um scanner em escala de cinza que fornecia uma imagem pixelizada com o tom de cinza observado para comparar o estado da amostra antes e depois do carregamento de três horas no MMLS3. 0 principal problema observado com essa metodologia era que o scanner identificava além da cor, a textura das amostras, além de ser afetada pelo reflexo de luz durante o ensaio, podendo confundir sombreamento e texturas mais rugosas com excesso de ligante.

Chaturabong (2014) adaptou o ensaio do Loaded Wheel Test (LWT) realizado na dosagem de MRAF 
para amostras de TSP, a fim de avaliar a exsudação, e utilizou como ferramenta de avaliação o software Image Processing \& Analysis System 2 (iPas 2), desenvolvido pelo Modified Asphalt Research Center (MARC). Já Adams (2014) fez o processamento com auxílio do software Matlab, no qual se identificaram as áreas pretas (exsudadas, com excesso de emulsão) e comparou-se com a área total da amostra. A proporção entre a área preta e a área branca gerou um percentual de exsudação na amostra.

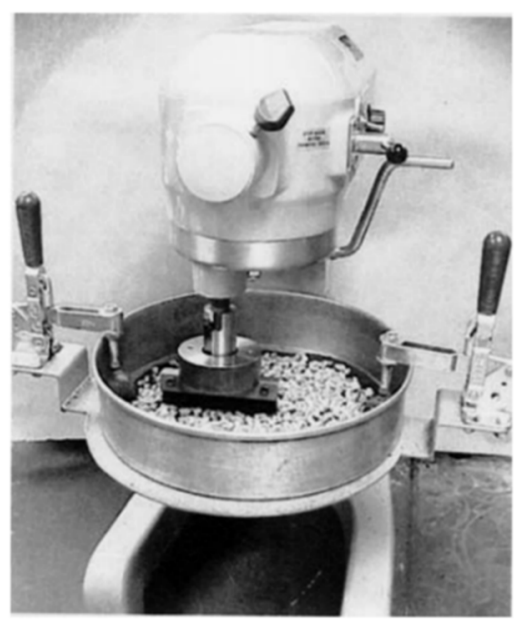

(a) Fonte: ASTM D7000 (2011).

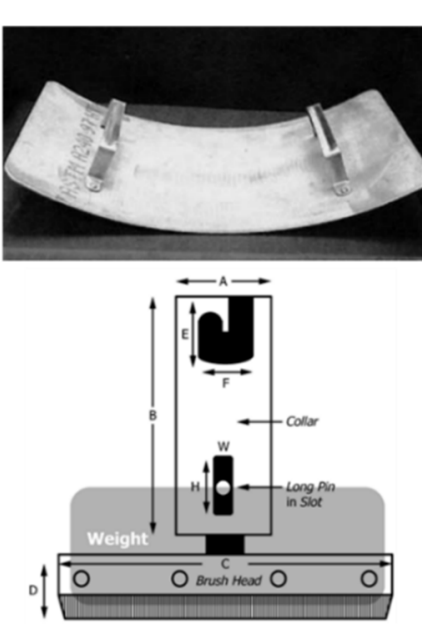

Figura 3: Equipamentos para realização do Flip-Over Test (a) e do Vialit Test (b).

\subsection{Considerações sobre o PUC}

Os agregados mais adequados para emprego em TSPs devem ter as dimensões as mais semelhantes possíveis, pois o processo construtivo é realizado em campo e não é possível garantir uma homogeneidade do revestimento em caso de agregados de tamanhos muito diferentes. Os agregados menores poderiam se "afogar" no ligante aplicado e os agregados maiores poderiam se soltar facilmente do revestimento devido a camada fina de ligante (TRANSIT NEW ZEALAND, 2005).

Tendo em vista a importância da escolha do agregado, Lee e Kim (2008b) definiram um parâmetro teórico chamado de PUC, o coeficiente de uniformidade baseado no desempenho, do inglês PerformanceBased Uniformity Coefficient (PUC). 0 coeficiente PUC fornece um indicativo de qualidade do agregado para emprego em tratamentos superficiais (chamados no estado da Carolina do Norte de chip seals) pela análise da sua curva granulométrica.

O PUC une o critério de falha de TSPs de McLeod (Figura 4) e o coeficiente de uniformidade utilizado para solos. De acordo com a sua teoria, o risco de falhas pelo excesso de ligante ou perda de agregado seria minimizado caso a graduação dos agregados fosse corretamente escolhida e a emulsão deveria preencher cerca de 70\% dos vazios entre as partículas de agregado do TSP para alcançar um bom desempenho (McLeod, 1969 apud Lee e Kim, 2009).

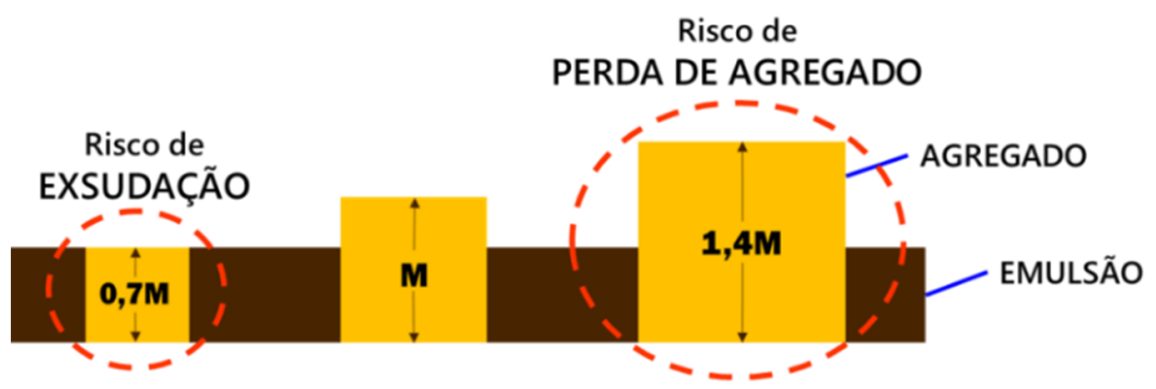

Figura 4: Critério de McLeod (Adaptado de Lee e Kim, 2009). 
Com base nos conceitos apresentados, a porcentagem de excesso de ligante e de perda de agregado pode ser calculada pelas Equações (1) e (2), respectivamente. Após vários estudos, equacionou-se o PUC como sendo função de duas variáveis, como pode ser observado na Equação (3).

Excesso de ligante $(\%)=\mathrm{P}_{\mathrm{EM}}$

$$
\text { Perda de agregado }(\%)=100-\mathrm{P}_{2 \mathrm{EM}}
$$

em que:

$$
\mathrm{PUC}=\frac{\mathrm{P}_{\mathrm{EM}}}{\mathrm{P}_{2 \mathrm{EM}}}
$$

- M é o diâmetro correspondente a 50\% do material que passa retirado diretamente da curva granulométrica do agregado, sendo assim a mediana da curva granulométrica;

- $\mathrm{P}_{\mathrm{EM}}$ é numericamente o percentual que passa, retirado da curva granulométrica do agregado, correspondente ao diâmetro $0,7 \mathrm{M}$. Representa a quantidade de agregado que apresenta tamanho muito menor que o da mediana e apresenta riscos de exsudação;

- $\mathrm{P}_{2 \mathrm{EM}}$ é o percentual que passa, retirado da curva granulométrica do agregado, correspondente ao diâmetro 1,4M. Representa a quantidade de agregado que apresenta tamanho muito maior que o da mediana e apresenta riscos de se soltar do revestimento.

Por definição, quão menor a área exsudada e menor a perda de agregado, melhor a qualidade do revestimento. Portanto, dado que o PUC é diretamente proporcional ao $\mathrm{P}_{\mathrm{EM}}$, quão menor for esse parâmetro melhor. Em contraponto, $\mathrm{P}_{2 \mathrm{EM}}$ maiores geram menor porcentagem de perda de agregado e menor PUC, logo quanto maior for esse parâmetro melhor. Lee e Kim (2009) concluíram que quanto menor o PUC, mais adequado para aplicação em serviços de TSP é o agregado. No estudo, encontrou-se uma relação entre a perda de agregado calculada para diferentes curvas granulométricas e a perda de agregado após o carregamento do simulador de tráfego MMLS3 e foi possível encontrar uma equação matemática (4) para o agregado ensaiado.

Perda de agreg. MMLS3 (\%) = 1,2922 + 0,2691 $\times$ perda de agreg.calculada (\%)

Observa-se que vários trabalhos internacionais (Lee e Kim, 2009; Transit in New Zealand, 2005) escolhem a melhor granulometria dos agregados com base na sua uniformidade. Sendo assim, é importante investigar como se esse conceito é considerado nas normas técnicas nacionais. Relatos de campo dos técnicos do DER/CE tem mostrado que, no geral, os revestimentos em TSP dosados pela especificação do DER (DERT-ES-P 10/00) têm apresentado rejeições menores do que quando investigados à luz da especificação do DNIT (ES 146-12). Essa constatação pode estar relacionada com a não exigência de uniformidade dos agregados no método do DNIT, como pode ser verificado pelas faixas explicitadas na DNIT 146/2012 - ES. Já o DER-CE, mesmo que não apresente exigência direta, recomenda agregados monogranulares (com diâmetros máximo e mínimo próximos) e apresenta faixas mais restritas.

\section{MATERIAIS E MÉTODOS}

A fim de introduzir o conceito de PUC nos estudos de TSP no Brasil, procurou-se inicialmente compreender a importância da uniformidade para o desempenho desse tipo de revestimento e verificar como é abordada a escolha dos agregados nas especificações técnicas do DNIT e do DER-CE. Após essas etapas, foram analisados dois agregados do estado do Ceará, calculou-se o PUC de cada um deles e foi verificado se havia alguma correlação entre a uniformidade e os resultados obtidos no ensaio de desgaste WTAT para avaliação do desempenho de TSPs adaptado por Loiola (2009) e modificado por Pereira (2010, 2013).

\subsection{Cálculo do PUC}

Inicialmente, para a obtenção do PUC das faixas do DNIT e das amostras de agregado em estudo, adotouse o seguinte procedimento: 
1. Realizaram-se os ensaios de granulometria por peneiramento de acordo com a DNER-ME 083/98 e traçou-se a curva de distribuição granulométrica de cada agregado;

2. Para cada uma delas, identificou-se a mediana da curva correspondente a $50 \%$ de material passante $\left(\mathrm{D}_{50}\right)$;

3. Utilizou-se o princípio de McLeod e calcularam-se os tamanhos de agregado mínimo $(0,7 \times \mathrm{M})$ e máximo $(1,4 \times M)$ desejáveis. Sendo assim, traçaram-se retas verticais até cruzar com a curva granulométrica;

4. Identificou-se o percentual com risco de exsudação $\left(\mathrm{P}_{\mathrm{EM}}\right)$ e com risco de desagregação $\left(\mathrm{P}_{2 \mathrm{EM}}\right)$ (Equações 1 e 2) pelo traçado de retas horizontais a partir da intersecção das retas verticais traçadas anteriormente com a curva granulométrica;

5. Calculou-se o PUC conforme a Equação 3, tendo-se os valores obtidos de $\mathrm{P}_{\mathrm{EM}}$ e $\mathrm{P}_{\text {2EM. }}$.

\subsection{Avaliação dos agregados recomendados em normas técnicas do DNIT}

Após a compreensão do conceito de PUC, obtiveram-se os limites das faixas do DNIT para TSS de acordo com a ES 146-12 Pavimentação asfáltica - Tratamento superficial simples e calculou-se o PUC dos limites da Faixa A e da Faixa B, a fim de verificar se apresentavam uniformidade satisfatória. Apesar das suspeitas que o procedimento do DER/CE poderia apresentar melhores resultados, não foi possível avaliar o parâmetro PUC, pois na especificação de serviço DERT-ES-P 10/00 não é proposta uma faixa granulométrica como a especificada pelo método do DNIT.

\subsection{Coleta de dados dos agregados do estado do Ceará}

Por fim, coletaram-se dados de pesquisas (ver Pereira, 2013) que utilizaram o método do DER/CE para determinação da granulometria do agregado e verificação do desgaste de TSS pelo procedimento WTAT. Sendo assim, no presente trabalho foram investigados dois agregados de origens minerais diferentes empregados em TSS, sendo um da pedreira Pyla proveniente de um gnaisse e o outro da pedreira OCS proveniente de um fonolito. 0 ligante empregado para preparação das amostras de TSS foi a emulsão asfáltica RR-2C.

\section{APRESENTAÇÃO E DISCUSSÃO DOS RESULTADOS}

\subsection{PUC das faixas do dnit para tratamento superficial simples}

Para verificação do PUC das faixas granulométricas do DNIT recomendadas para emprego em TSS (Tabela 1), avaliou-se a curva granulométrica dos limites inferiores e superiores de cada uma delas. Os traçados auxiliares ao cálculo do PUC das curvas dos limites inferior e superior da Faixa A e da Faixa B podem ser observados nos gráficos das Figuras 5 e 6, respectivamente.

Tabela 1: Granulometria dos agregados para aplicação em TSS.

\begin{tabular}{ccccc}
\hline \multicolumn{2}{c}{ Peneiras } & \multicolumn{2}{c}{ Faixas } & \multirow{2}{*}{ Tolerância da faixa de projeto } \\
\cline { 1 - 4 } Polegadas & $\mathbf{m m}$ & A & B & \\
\cline { 1 - 3 } 1/2" & 12,7 & 100 & - & \pm 7 \\
$3 / 8 "$ & 9,5 & $85-100$ & 100 & \pm 7 \\
№ 4 & 4,8 & $10-30$ & $85-100$ & \pm 5 \\
№ 10 & 2,0 & $0-10$ & $10-40$ & \pm 5 \\
№ 200 & 0,074 & $0-2$ & $0-2$ & \pm 2 \\
\hline Fonte: DNIT 146/2012- ES. & & &
\end{tabular}

Na Tabela 2 organizaram-se os parâmetros encontrados nas curvas apresentadas nas Figuras 5 e 6. Avaliando os valores de PUC obtidos para a Faixa A, observaram-se valores entre 0,179 e 0,343. Para a Faixa B, obtiveram-se valores de PUC entre 0,231 e 0,493, aproximadamente. O PUC do limite superior 
de ambas as faixas é considerado alto, pois para Lee e Kim (2009) o ideal é que ele seja o menor possível (o mais próximo de zero), indicando que agregados muito próximos deste limite, mesmo ainda satisfazendo a curva exigida pelo DNIT, podem apresentar altos índices de perda de agregado ou exsudação. Ao analisar os demais parâmetros, percebe-se um valor alto de $\mathrm{P}_{\mathrm{EM}}$, indicando a exsudação como crítica nos limites superiores de ambas as faixas.
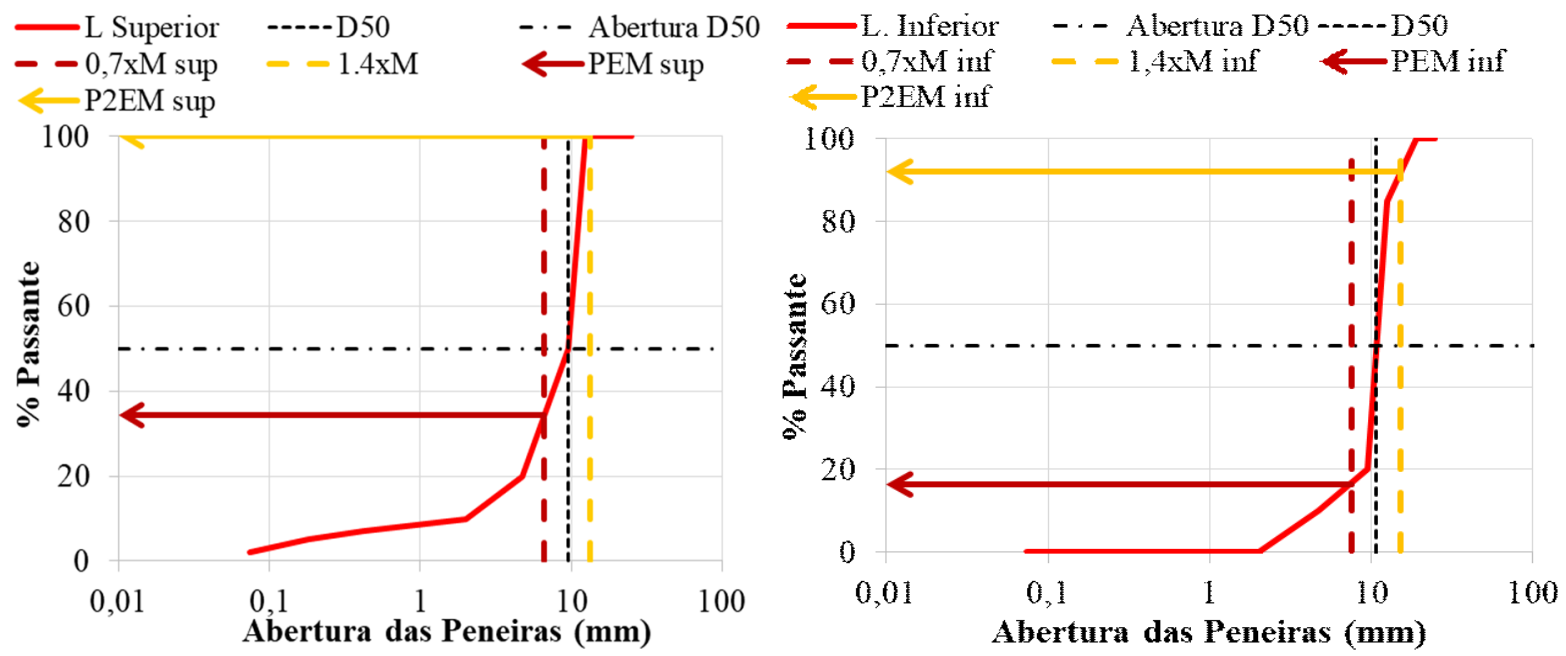

Figura 5: Traçados da Faixa A do DNIT para TSS para obtenção do PUC
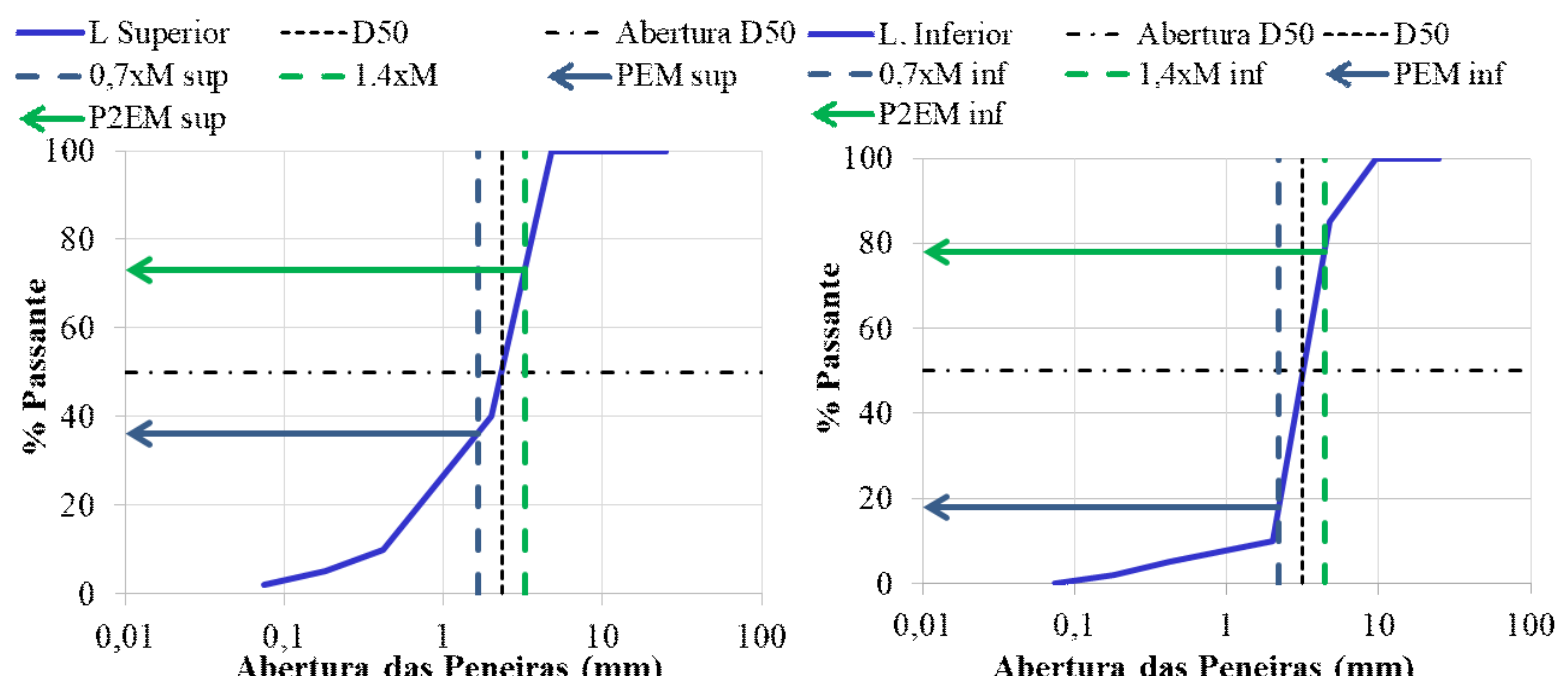

Figura 6: Traçados da Faixa B do DNIT para TSS para obtenção do PUC

Tabela 2: PUC dos limites das faixas do DNIT para TSS.

\begin{tabular}{|c|c|c|c|c|c|c|c|c|c|c|}
\hline \multicolumn{5}{|c|}{ FAIXA A } & \multicolumn{6}{|c|}{ FAIXA B } \\
\hline \multirow{3}{*}{ Limite superior } & $0.7 \times \mathrm{M}$ & 6,65 & & $P_{E M}(\%)$ & 34,3 & $0.7 \times M$ & 1,65 & & $P_{E M}(\%)$ & 36 \\
\hline & $M$ & 9,50 & $\mathrm{~mm}$ & $\mathrm{P}_{2 \mathrm{EM}}(\%)$ & 100 & M & 2,35 & $\mathrm{~mm}$ & $P_{2 E M}(\%)$ & 73 \\
\hline & $1.4 \times \mathrm{M}$ & 13,30 & & $P_{\text {UUP }}$ & 0,343 & $1.4 \times \mathrm{M}$ & 3,29 & & PUC $_{\text {SUP }}$ & 0,493 \\
\hline \multirow{3}{*}{ Limite inferior } & $0.7 \times \mathrm{M}$ & 7,56 & & $\mathrm{P}_{\mathrm{EM}}(\%)$ & 16,5 & $0.7 \times \mathrm{M}$ & 2,21 & & $P_{\mathrm{EM}}(\%)$ & 18 \\
\hline & $M$ & 10,80 & $\mathrm{~mm}$ & $P_{2 E M}(\%)$ & 92 & $M$ & 3,15 & $\mathrm{~mm}$ & $P_{2 E M}(\%)$ & 78 \\
\hline & $1.4 \times \mathrm{M}$ & 15,12 & & $\mathrm{PUC}_{\mathrm{INF}}$ & 0,179 & $1.4 \times \mathrm{M}$ & 4,41 & & $\mathrm{PUC}_{\mathrm{INF}}$ & 0,231 \\
\hline
\end{tabular}

O PUC do limite inferior da Faixa B é maior que o da Faixa A, apesar de não apresentar valores tão elevados quanto os dos limites superiores. Portanto, com base nas recomendações de Lee e Kim (2009), agregados de granulometria semelhante ao limite inferior da faixa A seriam os mais recomendados ao emprego em TSS, pois apresentam menor PUC e por consequência melhor previsão de desempenho. 


\subsection{Análise dos agregados do Ceará: dados de granulometria, caracterização e dosagem}

Os dados utilizados neste item se referem aos resultados obtidos por Pereira (2013) e coletaram-se os dados de caracterização (Tabela 3) dos agregados referentes à dosagem e perda de agregado no WTAT (Tabela 4), bem como dados de granulometria (Tabela 5). Os valores aqui dispostos são valores médios, extraídos da moldagem de 9 corpos de prova para cada tipo de agregado, cujas análises estatísticas completas podem ser verificadas na fonte citada.

Tabela 3: Resultados dos ensaios de caracterização dos agregados (Pereira, 2013).

\begin{tabular}{ccc}
\hline PARÂMETROS & Agregado 1 & Agregado 2 \\
\hline \% Passante \#200 & 0,24 & 0,21 \\
Abrasão Los Angeles (\%) & 36,59 & 18,33 \\
Índice de forma & 0,87 & 0,94 \\
Durabilidade & 1,7 & 2,4 \\
Densidade & 2,7 & 2,5 \\
Absorção (\%) & 0,6 & 0,4 \\
\hline
\end{tabular}

Tabela 4: Dados de perda dos agregados no WTAT e de dosagem (Pereira, 2013).

\begin{tabular}{|c|c|c|c|}
\hline & & TSS Agregado 1 & TSS Agregado 2 \\
\hline \multirow{2}{*}{ WTAT } & Desgaste médio & $7,81 \%$ & 11,5 \\
\hline & $14,79 \%$ & Desvio padrão & 14,63 \\
\hline \multirow{4}{*}{ Agregado } & & & Fonolito \\
\hline & Urigem & Gnalsse & DER-CE \\
\hline & $\begin{array}{l}\text { Teor 1a camada } \\
\text { Tetodo dosagem }\end{array}$ & $\begin{array}{l}\text { DER-CE } \\
14,39 \mathrm{~kg} / \mathrm{m}^{2}\end{array}$ & $\mathrm{~kg} / \mathrm{m}^{2}$ \\
\hline & & & $13,82 \mathrm{~kg} / \mathrm{m}^{2}$ \\
\hline \multirow{2}{*}{ Emulsão } & Teor 1a camada & $0,9 \mathrm{~L} / \mathrm{m}^{2}$ & $0,9 \mathrm{~L} / \mathrm{m}^{2}$ \\
\hline & Banho diluído & $0,3 \mathrm{~L} / \mathrm{m}^{2}$ & $0,3 \mathrm{~L} / \mathrm{m}^{2}$ \\
\hline
\end{tabular}

Tabela 5: Resumo dos percentuais passantes (Pereira, 2013).

\begin{tabular}{cccc}
\hline \multicolumn{2}{c}{ PENEIRAS } & \multicolumn{2}{c}{ \% QUE PASSA } \\
\hline Pol & $\mathbf{m m}$ & Agregado 1 & Agregado 2 \\
\hline 1" & 25,4 & 100,00 & 100,00 \\
3/4" & 19,1 & 92,50 & 80,81 \\
1/2" & 12,5 & 21,94 & 19,04 \\
3/8" & 9,5 & 3,64 & 7,87 \\
№ 4 & 4,76 & 1,03 & 2,21 \\
№ 10 & 2 & 0,99 & 1,26 \\
№ 200 & 0,074 & 0,92 & 0,68 \\
Fundo & - & 0,66 & 0,41 \\
\hline
\end{tabular}

\subsection{PUC dos agregados de Pereira (2013)}

Para dar início ao cálculo do PUC, traçou-se a curva granulométrica dos agregados a partir dos dados contidos na Tabela 6 e em seguida continuou-se o processo para obtenção dos demais parâmetros. Os valores obtidos para os parâmetros $0,7 \times \mathrm{M}, 1,4 \times \mathrm{M}, \mathrm{P}_{\mathrm{EM}}, \mathrm{P}_{2 \mathrm{EM}}$ e PUC de cada agregado foram obtidos graficamente com base no traçado da curva granulométrica. Os parâmetros calculados foram organizados na Tabela 6, bem como os dados referentes à perda de agregado no WTAT e perda teórica (100 - $\left.\mathrm{P}_{2 \mathrm{EM}}\right)$ proposta por Lee e Kim (2009). Em seguida, elaborou-se o gráfico da Figura 7 com os dados da PUC dos dois agregados e perda obtida no ensaio WTAT. Verificando-se o valor do PUC dos agregados minerais, perceberam-se valores muito próximos, já que apresentam granulometrias semelhantes.

Avaliando os valores de perda teórica (Figura 7), percebeu-se que ambos os agregados apresentaram PUC baixos (mais próximos de zero) e perda no WTAT satisfatória, pois pela recomendação de Pereira (2013) desgastes inferiores a 20\% são classificados como sendo de bom desempenho. Entretanto, observou-se que o agregado com menor PUC não foi o que apresentou menor desgaste no ensaio WTAT. Tal 
resultado pode ser consequência de diversos fatores, como a origem diferente dos agregados (gnaisse e fonolito) ou a dosagem escolhida, pois a uniformidade do agregado não é o único fator de influência a ser analisado. Outra possibilidade pode ser a ocorrência de problemas no ensaio proposto, cujos valores de desvio padrão foram considerados altos, indicando problemas na repetibilidade do ensaio.

Tabela 6: Resumo dos valores obtidos de PUC e demais parâmetros.

\begin{tabular}{ccc}
\hline Parâmetros & TSS Ag 1 & TSS Ag 2 \\
\hline 0,7×M & 10,40 & 10,85 \\
$1,4 \times \mathrm{M}$ & 20,79 & 21,70 \\
$\mathrm{P}_{\mathrm{EM}}(\%)$ & 9,5 & 13,0 \\
$\mathrm{P}_{2 \mathrm{EM}}(\%)$ & 94,5 & 89,7 \\
$100-\mathrm{P}_{2 \mathrm{EM}}(\%)$ & 5,5 & 10,3 \\
PUC & 0,101 & 0,145 \\
Taxa de emulsão $\left(\mathrm{L} / \mathrm{m}^{2}\right)$ & 1,20 & 1,20 \\
Taxa de agregado $\left(\mathrm{kg} / \mathrm{m}^{2}\right)$ & 14,39 & 13,82 \\
Origem do agregado & Gnaisse & Fonolito \\
Perda de agregado no WTAT & $14,8 \%$ & $7,8 \%$ \\
\hline
\end{tabular}

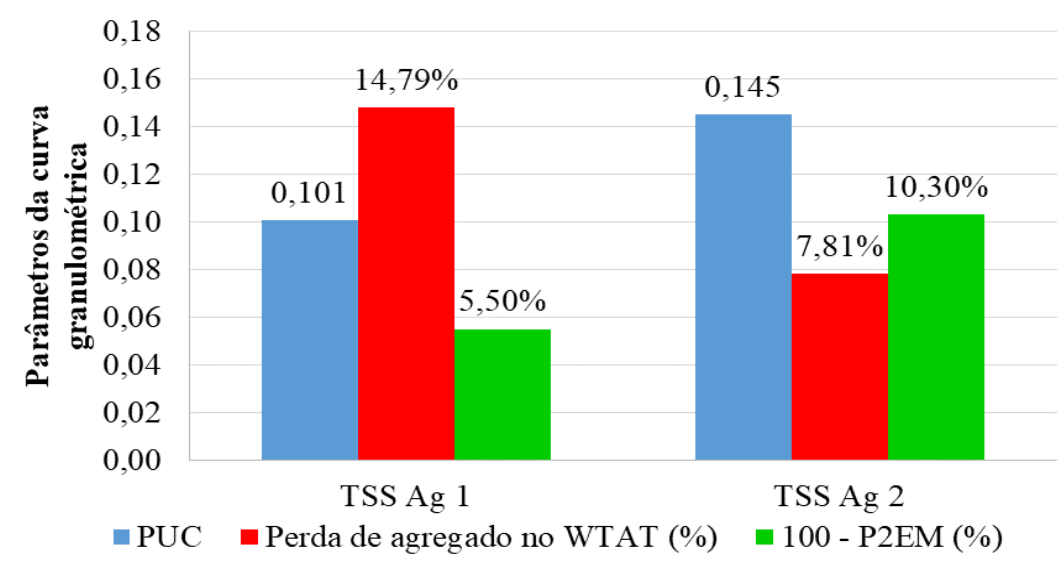

Figura 7: Gráfico comparativo do PUC, da perda teórica e da perda de agregado no WTAT

Analisando-se a relação entre a perda teórica e o desgaste obtido no MMLS3 (Equação 4), percebe-se que o ensaio WTAT é bem mais severo, pois no estudo de Lee e Kim (2009) as perdas calculadas eram maiores que as obtidas no simulador, enquanto que no WTAT o desgaste observado é bem superior ao calculado.

A severidade superior do ensaio WTAT em relação ao empregado por Lee e Kim (2009) pode ter superestimado os valores de perda. Além disso, existem aspectos associados com a execução dos ensaios, tais como compactação não uniforme ou insuficiente, as condições de temperatura, ou até mesmo demora no processo, que afetaria a interação entre ligante e agregado. Apesar das hipóteses citadas, acredita-se que o ensaio pode ser empregado para verificação de um parâmetro limite, como o sugerido por Pereira (2013), de valor de perda máximo de $20 \%$.

Além disso, as discrepâncias nos resultados obtidos em comparação aos de Lee e Kim (2008b) poderiam também ser justificadas pela diferença de tamanho médio dos agregados empregados. Pereira (2013) empregou no seu TSS um agregado de tamanho médio próximo a 3/4", enquanto que Lee e Kim (2009) mais próximo a 1/4" (Figura 8). 0 agregado mais graúdo de Pereira poderia ser mais suscetível ao arrancamento pelo tráfego do que o estudado por Lee e Kim (2008b). 


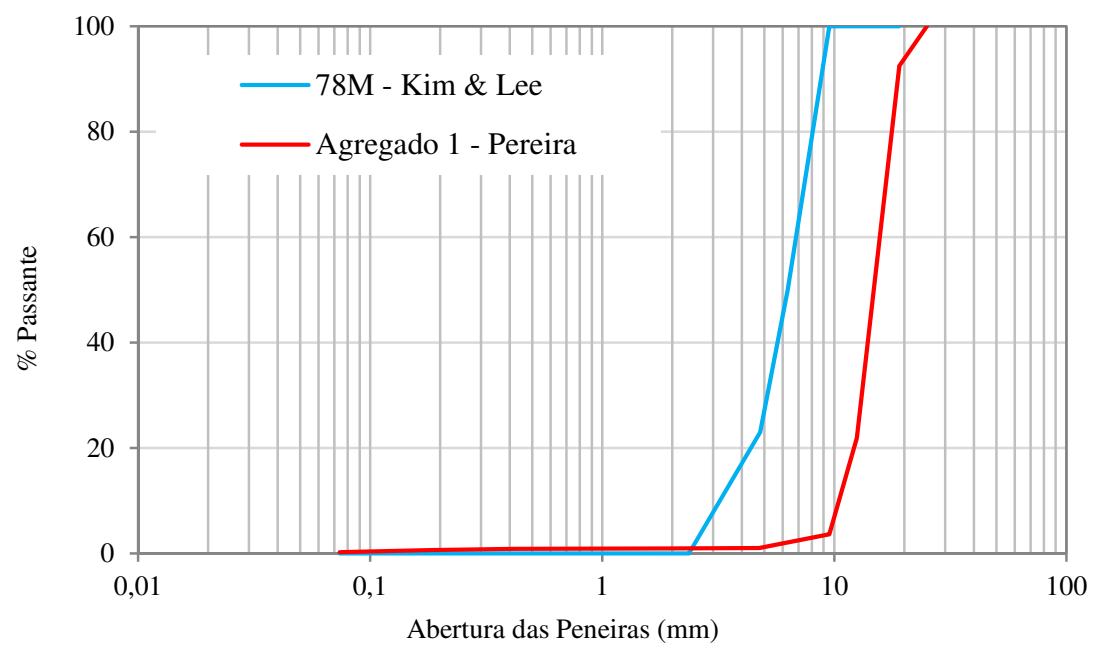

Figura 8: Tamanho dos agregados de Lee e Kim (2008b) e Pereira (2013).

\section{CONSIDERAÇÕES FINAIS}

Observou-se pela bibliografia consultada que o parâmetro PUC é um bom indicativo de qualidade para seleção de agregados em serviços de TSP. No entanto, o desempenho final do revestimento irá depender simultaneamente de outros fatores, como a dosagem aplicada, o método construtivo e a compatibilidade entre ligante e agregado. Deve-se ressaltar que os tratamentos do tipo duplo e triplo ainda precisam ser avaliados à luz do PUC, já que até o momento as pesquisas desenvolvidas correlacionaram o parâmetro apenas com tratamentos superficiais simples.

Ao calcular o PUC das faixas recomendadas pelo DNIT para TSS, observaram-se algumas limitações. Percebeu-se que apenas agregados com granulometria semelhante ao limite inferior da faixa A seriam considerados ideais para esse tipo de revestimento. Teoricamente, o limite superior da faixa A e toda a faixa $B$ teriam grande tendência à exsudação. Conclui-se a partir disso que agregados pertencentes às faixas recomendadas pelo DNIT não necessariamente apresentarão bom desempenho quando empregados em TSPs. Recomenda-se que as faixas do DNIT sejam alvo de um programa experimental que comprove, ou não, as hipóteses aqui levantadas. 0 trabalho aqui apresentado contribui para que os órgãos rodoviários façam uma reflexão acerca da necessidade de mudança dessas faixas, pois à luz do parâmetro PUC as faixas do DNIT não são consideradas adequadas.

Já o procedimento estabelecido pelo DER-CE para TSP recomenda indiretamente a seleção de agregados mais uniformes e de menor PUC, o que pode conduzir a melhor dosagem e qualidade desse tipo de revestimento. Conforme relatos técnicos dentro e fora do estado, o DER-CE tem se destacado na construção de trechos de bom desempenho em TSP, embora ainda ocorram presença de perda de agregados e exsudação. No futuro, seria interessante incluir nas normas para tais serviços algum parâmetro semelhante ao PUC para evitar a presença dos defeitos mais críticos.

Não foi possível identificar uma correlação direta entre o PUC e a perda de agregado no WTAT, mas sim uma tendência de resultados, pois o banco de dados para estudo ainda é pequeno e os agregados investigados têm origem diferente. Apesar de não existirem normas para emprego do WTAT como ensaio de avaliação da perda de agregado dos TSPs, este ainda é o único método laboratorial considerado de baixo custo existente no estado do Ceará que pode mensurar esse parâmetro. Os simuladores de laboratório são considerados os equipamentos mais apropriados para essa análise, como por exemplo o MMLS3, no entanto seu custo de aquisição é bastante elevado.

Notou-se que o parâmetro PUC pode ser eficaz na seleção preliminar de agregados convencionais e alternativos, a fim de diminuir os riscos dos defeitos decorrentes da perda de agregado e da exsudação. Uma sugestão de estudo futuro seria o desenvolvimento de um método de dosagem que contemple agregados alternativos (como o fresado e o agregado siderúrgico, por exemplo) e validem a possibilidade de 
sua aplicação em serviços de TSPs. Devido às diferenças de propriedades físicas e químicas, acredita-se que as taxas propostas nas normas nacionais para agregados convencionais não necessariamente se adequam aos alternativos.

Outra necessidade é o aperfeiçoamento dos métodos de avaliação da perda de agregado, adaptando os ensaios normatizados internacionalmente ao contexto nacional e propondo limites aceitáveis aos órgãos reguladores. Também se recomenda o desenvolvimento de um método para avaliação da exsudação, defeito ainda não estudado no Brasil e essencial para um processo de dosagem mais eficiente. Enfim, após a consolidação das técnicas de laboratório, devem ser realizados estudos com a finalidade de encontrar uma relação entre os resultados de ensaio e o desempenho do revestimento no campo.

\section{AGRADECIMENTOS}

Os autores agradecem à Fulbright e à Capes pelo apoio financeiro para a realização da presente pesquisa, bem como aos pesquisadores da North Carolina State University.

\section{REFERÊNCIAS}

Adams, J. M. (2014) Development of a Performance-Based Mix Design and Performance-Related Specification for Chip Seal Surface Treatments. Tese (doutorado). Civil Engineering, North Carolina State University. Raleigh.

ASTM (2011) D7000 - 11 Standard Test Method for Sweep Test of Bituminous Emulsion Surface Treatment Samples. ASTM International. West Conshohocken.

Chaturabong, P. (2014) Development of the Loaded Wheel Tracking Test (LWT) for Evaluating Bleeding in Chip Seals and Relationship to Emulsion Residue Rheology. Tese (doutorado). Civil \& Environmental Engineering, University of Wisconsin. Madison.

CNT (2018) Boletim estatístico. Confederação Nacional do Transporte (CNT). Disponível em: <http://www.cnt.org.br/Boletim/boletim-estatistico-cnt>. (Acesso em: 02 março 2018).

DER-CE (2017). Informativo gerencial 2017. Disponível em: <http://www.der.ce.gov.br/wp-content/uploads/sites/30/2018/01/informativo_gerencial2017.pdf >. (Acesso em: 19/04/2018).

DNIT (2012) ES 146/2012 Pavimentação asfáltica - Tratamento superficial simples - Especificação de serviço. Rio de Janeiro.

DNIT (2006) Manual de Pavimentação. Publicação IPR - 719. Rio de Janeiro.

Larsen, J. (1985) Tratamento Superficial na Conservação e Construção de Rodovias. ABEDA. Rio de Janeiro.

Lee, J. S. e Kim, Y. R. (2010) Evaluation of Performance and Cost-Effectiveness of Polymer-Modified Chip Seals. Transportation Research Record: Journal of the Transportation Research Board, v. 2150, Transportation Research Board of the National Academies. Washington, D.C., p. 79-86. DOI: 10.3141/2150-10

Lee, J. S. e Kim, Y. R. (2009) Performance-Based Uniformity Coefficient of Chip Seal Aggregate. Transportation Research Record: Journal of the Transportation Research Board, v. 2108, Transportation Research Board of the National Academies, Washington, D.C., p. 53-60. DOI: 10.3141/2108-06

Lee, J. S. e Kim, Y. R. (2008) Understanding the Effects of Aggregate and Emulsion Application Rates on Performance of Asphalt Surface Treatments. Transportation Research Record: Journal of the Transportation Research Board, v. 2044, Transportation Research Board of the National Academies. Washington, D.C., p. 71-78. DOI: 10.3141/2044-08

Lee, J.; Kim, Y. (2010) Optimal Distribution of Rolling Coverage in Multiple Chip Seals. Transportation Research Record: Journal of the Transportation Research Board, v. 2150, p. 70-78. DOI: 10.3141/2150-09.

Loiola, P. R. R. (2009) Estudo de Agregados e Ligantes Alternativos para Emprego em Tratamentos Superficiais de Rodovias. Dissertação (mestrado). Departamento de Engenharia de Transportes, Universidade Federal do Ceará. Fortaleza.

Moraes, R.; Bahia, H. (2013) Effects of Curing and Oxidative Aging on Raveling in Emulsion Chip Seals. Transportation Research Record: Journal of the Transportation Research Board, v. 2361, n. 2361, p. 69-79. DOI: 10.3141/2361-09

Nogami, J. S.; Villibor, D. F. (1995) Pavimentação de Baixo Custo com Solos Lateríticos. Editora Villibor. São Paulo.

Pereira, S. L. O. (2010) Avaliação de Tratamentos Superficiais de Rodovias através de Análise de Laboratório. Monografia (iniciação científica). Departamento de Engenharia de Transportes, Universidade Federal do Ceará. Fortaleza.

Pereira, S. L. O. (2013) Avaliação dos Tratamentos Superficiais simples, duplo e triplo de rodovias através do emprego de diferentes agregados da região metropolitana de Fortaleza. Dissertação (mestrado). Departamento de Engenharia de Transportes, Universidade Federal do Ceará. Fortaleza.

Silva, R. C. (2016) Introdução de um novo coeficiente para análise de desempenho dos tratamentos superficiais a partir dos estudos desenvolvidos nos Estados do Ceará/Brasil e Carolina do Norte. Monografia (iniciação científica). Departamento de Engenharia de Transportes, Universidade Federal do Ceará. Fortaleza.

Transit New Zealand (2005) Chipsealing in New Zealand. ISBN: 0-478-10562-2. 